\title{
Theoretical study of the ionization of an alkali atom adsorbed on a metal surface by a laser-assisted subfemtosecond pulse
}

\author{
A. K. Kazansky ${ }^{1,2}$ and P. M. Echenique ${ }^{2,3}$ \\ ${ }^{1}$ Fock Institute of Physics, The University of St. Petersburg, St. Petersburg 198504, Russia \\ ${ }^{2}$ Donostia International Physics Center (DIPC), Paseo Manuel de Lardizabal, San Sebastián/Donostia, E-20018 Basque Country, Spain \\ ${ }^{3}$ Departamento de Fisica de Materiales and CFM, Centro Mixto CCSIC-UPV/EHU, Facultad de Ciencias Quimicas, UPV/EHU, \\ Apdo. 1072, San Sebastián/Donostia, 20080 Basque Country, Spain
}

(Received 23 September 2009; revised manuscript received 20 November 2009; published 26 February 2010)

\begin{abstract}
A numerical simulation of the process of ionization of an atom adsorbed on a metal surface by the laserassisted subfemtosecond pulse is presented. The streaking scheme is considered, when a weak subfemtosecond pulse comes together with a strong IR pulse with a variable delay between them. The process is simulated by solving numerically the nonstationary Schrödinger equation in the cylindrical coordinate system. The double differential cross-sections (DDCS) are computed for a fixed delay between the pulses, but for few different carrier frequencies of the subfemtosecond pulse. The results obtained are compared with those in the gas phase. Beside the conventional streaking shift in energy, the surface influence on the DDCS is revealed. It is shown that the corresponding variation in DDCS noticeably depends on the frequency of the subfemtosecond pulse.
\end{abstract}

DOI: 10.1103/PhysRevB.81.075440

PACS number(s): 79.20.Ds, 78.47.D-

\section{INTRODUCTION}

The development of the subfemtosecond experimental technique initiated in 2001 (Ref. 1) provides a tool for monitoring electrons with subatomic resolution in both space and time, creating new opportunities for real-time observation and control of atomic-scale electron dynamics. ${ }^{2}$ By keeping track of the temporal evolution of the outgoing electron wave packets, this technique gives direct time-domain insight into various aspects of the interaction of an electron with very fast relaxing many-electron systems. The attosecond streaking spectroscopy has already enabled observation of the decay of an inner-shell vacancy through Auger relaxation in isolated atoms in the gas phase ${ }^{3-6}$ and led to the creation of attosecond chronoscopy. ${ }^{7-9}$ In most of the experiments, the time dynamics has been observed with an ingenious "streaking camera," 10,11 when the IR pulse, which produces the attosecond pulse by high-harmonics generation, is simultaneously used for transposing the electron ejection time into the electron energy spectrum. Although a large number of papers on attosecond physics ${ }^{12}$ have been already published, a very few proof-of-principle experiments have been performed. Meanwhile, comprehensive understanding of the dynamic behavior of electrons, especially in condensed-matter systems, is very important for promoting experimental investigations and their application in nanotechnology. The first experimental extension of the attosecond streaking technique to processes with solid surfaces has been done recently. ${ }^{13}$ This is a very important proof-of-principle experiment which has shown that it is possible to obtain direct time-domain access to charge dynamics at the near-surface region of metals by probing photoelectron emission from a single crystal.

In parallel with the experimental studies, development of theory, which should provide full understanding of the experimental findings, is of extreme importance. In general, the time-independent theoretical paradigm must be supplemented by a versatile time-dependent theory directly describing attosecond experiments in the time domain. At the cur- rent level of development of computational technique, it becomes possible to build up rather realistic models of the phenomena with atoms in the gas phase $\mathrm{e}^{9,14}$ which can be used for a step by step control of the processes. Regarding the processes with a solid surface involved, the theoretical simulations of the experiment ${ }^{13}$ have been presented in Ref. 15. Now it is urgent to develop the theoretical background for the description of the electron system of the solid-state interface under the joint action of the attosecond extreme ultraviolet XUV pulse and femtosecond pulse of strong IR field. This implies building up a set of versatile computational codes which, being based on an explicitly given Hamiltonian, allows one to simulate various processes studied experimentally. Of principal interest are investigations of various processes with surface impurities, including charge transfer, charge screening, image charge creation and decay, etc. The simplest object for such theoretical studies is a single adsorbate on a solid surface. The only quantity which can be measured in the attosecond experiments is the double differential (in the ejected electron energy and direction of its momentum) cross-section (DDCS). The general goal of the theoretical simulations of the processes is a direct computation of this quantity. This would provide a concise link between the observed data and the theoretical models and would allow one to extract from these data information on the dynamics of fast processes in the electron system at the surface of a solid.

\section{DESCRIPTION OF THE PROBLEM}

Here we present the first theoretical simulation of a streaking experiment with an atom adsorbed on a metal surface that can be of importance in planning the experiments. We consider a solitary alkali atom adsorbed on a noble metal surface. Alkali adsorbates are practically fully ionized on these surfaces, so that initially the adsorbate is in the positive-ion state. The attosecond pulse and the few femtosecond strong IR laser pulse are applied to the system. The 
attosecond pulse ionizes the adsorbate, transferring it to the state of doubly charged positive ion. The ejected electron moves in the fields of the adsorbate, the metal surface (including the ejected electron image charge), and the IR laser pulse field. Action by the IR field leads to the streaking effect which has been used to extract information on the time development of the processes. It is worth noting that the streaking effect itself is a rather robust phenomenon which can be obtained within the strong field approximation (SFA). This approximation gives rather good results, although they do not fully coincide with the results obtained with accurate computations. ${ }^{14}$ When the streaking effect is used to study processes in the time domain, very tiny deviations from the streaking effect are analyzed (see, e.g., Ref. 13). Therefore, our theoretical simulation, when applied to the analysis of the experimental data, has to be adjusted to the experiment under consideration. One more generic feature of the process is related to reflection of the ejected electron wave packet from the metal surface. It causes some interference, which also can be addressed experimentally.

In what follows we consider a solitary $\mathrm{Na}$ adsorbate placed at $Z_{a d}=2.5$ a.u. from the image plane of $\operatorname{Ag}(100)$, which is at a distance of $z_{i m}=2.064$ a.u. (Ref. 16) from the outermost layer of the metal toward the vacuum. The adsorbate is initially ionized to $\mathrm{Na}^{+}$state. Then, it is ionized once more from the $2 p$ shell by the XUV attosecond pulse (duration of about 200 as; we consider the pulses with photon energies 50 and $90 \mathrm{eV}$ ). The ejected electron moves in the potential $U_{2}(r)$ of the $\mathrm{Na}^{++}$ion, in the field of the lattice $U_{s}(z)$, and in the field of a relatively strong IR pulse with $\omega_{\mathrm{IR}}=1.6 \mathrm{eV}$. Both the light pulses are assumed to be polarized perpendicular to the surface. In this case, the component of the angular momentum of the ejected electron perpendicular to the surface is a good quantum number. The ionization can proceed from both $\sigma$ and $\pi$ states, strongly localized in the vicinity of the Na nucleus. In this work we study electron ejection close to the surface normal surface and restrict ourselves to the $\sigma$ case.

The basic Schrödinger equation governing the time evolution of the system reads

$$
\begin{aligned}
\imath \frac{\partial}{\partial t} \Psi(\vec{r}, t)= & {\left[\mathbf{H}\left(\vec{r}-\vec{R}_{a t}\right)-\imath \frac{\gamma(z)}{2}-E\right] \Psi(\vec{r}, t)+\left[U_{s}(z)\right.} \\
& \left.+E_{\mathrm{IR}}(z) \varepsilon_{\mathrm{IR}}(t) \cos \left(\omega_{\mathrm{IR}} t\right)\right] \Psi(\vec{r}, t) \\
& +\frac{1}{2} E_{\mathrm{XUV}}(t) z \Phi_{2 p}\left(\vec{r}-\vec{R}_{a t}\right)
\end{aligned}
$$

The Hamiltonian $\mathbf{H}\left(\vec{r}-\vec{R}_{a t}\right)$ describes the interaction of the electron with the $\mathrm{Na}^{++}$core; the corresponding potential has been computed with the Hartree-Slater approximation. The (pseudo)potential $U_{s}(z)$ in Eq. (1) reproduces the interaction of an electron with the lattice in a finite metal. For this potential, we use the parametrization by Chulkov et al. ${ }^{16}$ with parameters for the $\operatorname{Ag}(100)$ surface. The potentials given in that paper describe the electron motion in various metals in directions perpendicular to different surfaces of the metals. These potentials nicely reproduce the corresponding band structures, surface, and image states. In our problem, these structures can be important when the ejected electron energy is close to the threshold. By taking these structures in consideration, we make the code more versatile. The damping function $\gamma(z)$ in Eq. (1) takes into account electron-electron collisions in the bulk; it is nonzero at $z<0$. The quantity $\gamma(z)$ is approximated as $\sqrt{2 E} / \lambda_{f}$, where $\lambda_{f}$ is the mean free path of electron in the metal. It weakly depends on the electron energy, being close to $5 \AA$ at a few tens electron Volt range. ${ }^{17}$ We have set $\lambda_{f}=10$ a.u.

Since the XUV field is rather weak, the interaction between the active electron and the XUV attosecond pulse is described within the rotating wave approximation (RWA, for some details, see Refs. 9 and 14). The quantity $E$ in Eq. (1) is a sum of the electron energy in the initial state, $-\left|\bar{\epsilon}_{2 p}\right|$, and the carrier frequency of the XUV pulse $\omega_{\mathrm{XUV}}$. The RWA is a quite advantageous technical method, since the fast oscillations of the XUV field are excluded and therefore propagation of the wave packet can be performed with a reasonable time step. Within RWA, neither frequency nor intensity of the XUV field enters the present consideration. For correct application of RWA, the wave function of the active electron in the initial state has to be an eigenfunction of the Hamiltonian. This wave function $\Phi_{2 p_{\sigma}}\left(\vec{r}-\vec{R}_{a t}\right)$ has been precalculated taking into account the polarization of this state by the surface potential. The wave function is slightly changed by this interaction, but it strongly influences the energy of the initial state: $\bar{\varepsilon}_{2 p}=-1.392$ a.u., while the energy of the corresponding state for a single ion $\mathrm{Na}^{+}$within our model is $\varepsilon_{2 p}$ $=-1.690$ a.u. (The experimental value of this energy is $\varepsilon_{2 p}^{\text {exp }}=-1.738$ a.u.). The energy shift of the adsorbate state is close to the classical image shift $+3 / 4 Z_{a d}=0.3$ a.u. of the energy for an electron localized on the adsorbate center. The source term $1 / 2 E_{\mathrm{XUV}}(t) z \Phi_{2 p_{\sigma}}\left(\vec{r}-\vec{R}_{a t}\right)$, in Eq. (1) describes the ionization of the initial electron state by the XUV pulse with the envelope $E_{\mathrm{XUV}}(t)$. The interaction with the electromagnetic field is taken in the length form, the factor $1 / 2$ is due to the RWA used. For the envelope, we have used the form $\epsilon_{X}(t)=\exp \left[-\left(t-t_{\text {delay }}\right)^{2} / \tau_{X}^{2}\right]$ with $\tau_{X}=125$ as, full width at half maximum $(\mathrm{FWHM})=0.21$ fs.

In Eq. (1), the function $E_{\mathrm{IR}}(z)$ gives the $z$ dependence of the normal component of the IR electric field. This dependence deserves some discussion. First, we note that it is well known that a stationary electric field is fully screened in a metal with a screening length of about one atomic layer. If the electric field is slowly varying with time, the electron system follows it adiabatically and the screen length changes weakly. ${ }^{18}$ The character of the screening changes when the frequency of the external field approaches the surface plasmon frequency. In our case the IR field frequency $\omega_{\text {IR }}$ $=1.6 \mathrm{eV}$ is smaller than the surface plasmon frequency of the $\operatorname{Ag}(100) \omega_{\mathrm{Ag}(100)}^{s p}=3.7 \mathrm{eV} .{ }^{19}$ Therefore we assume that the screening length $\xi=4$ a.u. (Ref. 18) and take $E_{\mathrm{IR}}(z)$ as follows:

$$
E_{\mathrm{IR}}(z)= \begin{cases}\xi+\left(z-z_{i m}\right)+\kappa & z>z_{i m} \\ \xi \exp \left[\left(z-z_{i m}\right) / \xi\right]+\kappa & z<z_{i m}\end{cases}
$$

The parameter $z_{i m}$ in Eq. (2) gives the position of the image plane. It enters the parametrization of the pseudopotential 
$U_{s}(z)\left[\right.$ see, ${ }^{16}$ Eqs. (2)-(5)], for $\operatorname{Ag}(100) z_{\text {im }}=2.064$ a.u. As in the experiment, ${ }^{13}$ we assume that the streaking field is incident on the surface at the Brewster's angle $\left(\alpha_{B} \approx 78.5^{\circ}\right.$ for silver at the considered IR frequency). In this case, in the metal the component of the IR electric field perpendicular to the surface is $\kappa$ times weaker than the strength of the incident electric field $\left(\kappa=\cos ^{2} \alpha_{B} / \sin \alpha_{B},{ }^{20}\right.$ in our case $\left.\kappa \approx 0.04\right)$. The abrupt change in the perpendicular component of the IR electric field in the metal is a qualitative reason given in Ref. 15 for the time delay in ionization of the localized $4 f$ states with respect to the delocalized states revealed in Ref. 13. For the envelope of the IR pulse we use the form

$$
\epsilon_{\mathrm{IR}}(t)=0.5 \mathcal{E}_{0}\left\{1-\cos \left[\pi t / \tau_{\mathrm{IR}}\right]\right\}, \quad 0<t<2 \tau_{\mathrm{IR}}
$$

with the $\mathrm{FWHM}=\tau_{\mathrm{IR}}=5$ fs. The field strength $\mathcal{E}_{0}$ is related to the intensity of the IR pulse

$$
W_{\mathrm{IR}}=\frac{c}{8 \pi \tau_{\mathrm{IR}}} \int_{0}^{\tau_{\mathrm{IR}}} \epsilon_{\mathrm{IR}}^{2}(t) d t .
$$

We set $W_{\text {IR }}=10^{12} \mathrm{~W} / \mathrm{cm}^{2}$.

\section{A. Details of the computations}

The simulations of the processes with a single atom in the gas phase $\mathrm{e}^{9,14}$ are naturally performed in the spherical coordinate system. The components of the wave packet expanded over the spherical basis are mixed only by the IR field. The corresponding matrix in the Hamiltonian is a product of the radial coordinate and $r$-independent matrix. This allowed us to build up a rather effective computational scheme (see Refs. 9 and 14). As a rule, 40 partial waves are enough to reach convergence in those tasks. Importantly, the extraction of the physical results from the wave packet was performed by projecting the packet after its propagation till the IR pulse termination on the continuum eigenfunctions of the corresponding ion. If the laser pulse is longer than 10-20 fs, the propagation should continue quite long and, simultaneously, the radial mesh should become larger. Since the computations have been performed in the length gauge, the increase in the radial mesh requires a decrease in the time step. These requirements make the computation with this scheme unfeasible with an increase in the duration of the IR pulse.

In the present case the spherical coordinate system is not suitable for the computations. Since the wave packet promptly decreases in the half space $z<0$, in order to reach convergence one should include a large number of the partial waves while their mixing is not factorized. Besides, the continuum eigenfunctions of the system "adsorbate + surface" cannot be accurately calculated. Experience acquired in similar studies (see Ref. 21 and 22 and references therein) allows us to recommend the cylindrical coordinate system as more suitable for studying problems with adsorbates. However, one has to take into account the strong field of the nucleus in this coordinate system that causes a substantial technical problem. To deal with this problem, we have used nonuniform meshes (see Ref. 23 and 24) in both the $\rho$ and $z$ variables

$$
z=x-c \arctan (x / c), \quad \rho=y-c \arctan (y / c), \quad c=0.5
$$

with the uniform meshes in $x=(-620.5+k) \delta x, y=(j+1) \delta y$ with the steps $\delta x=0.13, \delta y=0.15$. Thus, both the meshes in $\rho$ and $z$ coordinates are very dense at the center of the adsorbate atom and uniform at large distance. This allows us to take into account the strong core Coulomb potential with high accuracy. In the present computations, the mesh covers the cylinder $z \in[-80,376], \rho \in[0,450]$ (in a.u.). The splitpropagation scheme has been used with a time step equal to 0.03 a.u. The outgoing wave asymptotic condition has been provided by the artificial absorbing potentials at the edges of the mesh. Propagation has been performed till $t_{\text {fin }}=\tau_{\mathrm{XUV}}$ +200 a.u. in the case of a XUV pulse with the frequency $\omega_{\mathrm{XUV}}=90 \mathrm{eV}$ and till $t_{\text {fin }}=\tau_{\mathrm{XUV}}+400$ a.u. in the case of $\omega_{\mathrm{XUV}}=45 \mathrm{eV}$. During this time the essential part of the wave packet can pass through the "moving registering detector" (see below), which has been placed, on average, at $z_{0}$ $=290$ a.u.

The computations in the cylindrical coordinate system cause a new problem. It is related to the procedure of extraction of physical quantities from the results of the timedependent computations. Here we put forward a different approach which develops the method described in Ref. 25. In the far zone, where only a time-dependent uniform electric field polarized along the $z$ axis acts on the electron, the basis functions, normalized at large time over the momentum scale, can be written as

$$
\psi_{\vec{q}, k_{z}}(\vec{\rho}, z, t)=\frac{1}{(2 \pi)^{3 / 2}} \exp \{l[\bar{Q}(t)+P(t) z+\vec{q} \vec{\rho}]\} .
$$

The Schrödinger equation

$$
l \frac{\partial}{\partial t} \psi_{\vec{q}, k_{z}}(\vec{\rho}, z, t)=\left[-\frac{1}{2} \Delta+\epsilon_{\mathrm{IR}}(t) z\right] \psi_{\vec{q}, k_{z}}(\vec{\rho}, z, t)
$$

leads to the explicit representation for $Q(t), P(t)$

$$
\begin{gathered}
P(t)=k_{z}+A(t) ; \quad A(t)=\int_{t}^{\infty} d \tau \epsilon_{\mathrm{IR}}(\tau), \\
\bar{Q}(t)=-\left(k_{z}^{2}+q^{2}\right) t / 2+k_{z} Z_{1}(t)+Z_{2}(t) / 2, \\
Z_{1}(t)=\int_{t}^{\infty} d \tau A(\tau) ; \quad Z_{2}(t)=\int_{t}^{\infty} d \tau A^{2}(\tau) .
\end{gathered}
$$

Here the quantity $A(t)$ is the vector potential of the IR field. The basis functions in Eq. (3) read

$$
\begin{aligned}
\psi_{\vec{q}, k_{z}}(\vec{\rho}, z, t)= & \frac{1}{(2 \pi)^{3 / 2}} \exp \left(l \left\{-\left(k_{z}^{2}+q^{2}\right) t / 2+k_{z}\left[z+Z_{1}(t)\right]\right.\right. \\
& \left.\left.+A(t) z+Z_{2}(t) / 2+\vec{q} \overrightarrow{1} \rho\right\}\right) .
\end{aligned}
$$

These functions merge with the conventional plane waves at $t=+\infty$. The expansion of any wave function 


$$
\Psi(\vec{\rho}, z, t)=\int_{0}^{\infty} d k_{z} d^{2} \vec{q} f\left(\vec{q}, k_{z}\right) \psi_{\vec{q}, k_{z}}(\vec{\rho}, z, t),
$$

which contains only outgoing waves, can be rewritten as

$$
\begin{aligned}
\Psi\left[\vec{\rho}, z_{0}-Z_{1}(t), t\right]= & e^{\imath\left\{A(t)\left[z_{0}-Z_{1}(t)\right]+Z_{2}(t) / 2\right\}} \\
& \times \int_{0}^{\infty} \frac{d k_{z} d^{2} \vec{q}}{(2 \pi)^{3 / 2}} e^{\imath\left[-\left(k_{z}^{2}+q^{2}\right) t / 2+k_{z} z_{0}+\vec{q} \vec{\rho}\right]} f\left(\vec{q}, k_{z}\right) .
\end{aligned}
$$

Thus, the amplitude $f\left(\vec{q}, k_{z}\right)$ is the Fourier transform of the wave packet value at the "moving screen" $z(t)=z_{0}-Z_{1}(t)$, where $z_{0}=z(\infty), \quad \epsilon=\left(k_{z}^{2}+q^{2}\right) / 2$

$$
\begin{aligned}
f\left(k_{z}, \vec{q}\right)= & (2 \pi)^{-3 / 2} k_{z} e^{i k_{z} z_{0}} \int_{-\infty}^{\infty} d t e^{l\left[\epsilon t-A(t) z(t)-Z_{2}(t) / 2\right]} \\
& \times \int d^{2} \vec{\rho} e^{-l \vec{q} \vec{\rho}} \Psi[\vec{\rho}, z(t), t] .
\end{aligned}
$$

Finally, the DDCS reads

$$
\begin{aligned}
\frac{d^{2} \sigma}{d \epsilon d \Omega}= & {\left[\frac{\sqrt{2 \epsilon}}{2 \pi}\right]^{3} \cos ^{2} \theta \mid \int_{-\infty}^{\infty} d t e^{\imath\left[\epsilon t-A(t) z(t)-Z_{2}(t) / 2\right]} } \\
& \times\left.\int d^{2} \vec{\rho} e^{-l \vec{q} \vec{\rho}} \Psi[\vec{\rho}, z(t), t]\right|^{2} \\
= & \frac{\cos ^{2} \theta}{2 \pi^{3} \sqrt{2 \epsilon}} \mid \int_{-\infty}^{\infty} d t e^{i \epsilon t} \frac{d}{d t}\left\{e^{\imath\left[-A(t) z(t)-Z_{2}(t) / 2\right]}\right. \\
& \left.\times \int d^{2} \vec{\rho} e^{-l \vec{q} \vec{\rho}} \Psi[\vec{\rho}, z(t), t]\right\}\left.\right|^{2} .
\end{aligned}
$$

Let compare effectiveness of the present method of extraction of information from the time-dependent computations with the method used before., ${ }^{9,14}$ Previously we have dealt with a spherically symmetrical atom in the fields of XUV and IR pulses. In that case the wave packet was propagated till the IR pulse termination and then it was projected on the continuum eigenstates which takes into account the electron scattering by the atom. In the present case that method cannot be applied. This is because after the pulse termination we have a system adsorbate + surface and the continuum eigenstates for this system cannot be computed. The present method of information extraction with the moving screen is the essential part of computations for such systems. The present approach has a noticeable advantage which can make it useful as well when a spherically symmetrical system is studied. Namely, with the method used previously, necessity of increase in size of the coordinate mesh makes the computations for IR pulses longer than 10-20 fs rather time consuming. Within the present approach, one should keep track of the wave-packet propagation only till it passes the moving detector. After that, the wave packet can be absorbed by the artificial potential. The only restriction on the mesh size now
TABLE I. Comparison of the ejected electron energies (in $\mathrm{eV}$ ) at maxima DDSC obtained in computations $\epsilon_{\max }$ and $\epsilon_{\text {eff }}$ obtained within the conventional theory of streaking.

\begin{tabular}{llllllll}
\hline \hline$\omega_{\mathrm{XUV}}$ & 75 & 80 & 85 & 90 & 95 & 53.1 & 98.1 \\
$\epsilon_{\max }$ & 31 & 35 & 41 & 46 & 51 & 5 & 46 \\
$\epsilon_{\text {eff }}$ & 31.6 & 36.2 & 40.9 & 45.5 & 50.2 & 4.82 & 45.5 \\
\hline \hline
\end{tabular}

is that the moving detectorz $(t)$ should not enter the region where the absorbing potential is operative. (Also, the "detector" should all the time be in the region where all fields, beside the uniform electric field, can be neglected.) This allows one to deal with rather long IR pulses. On the other hand, the present approach has some disadvantages. First, the computations have to be continued till the principal part of the wave packet passes through the detector, that is the computation time with this code is always longer than the run time of the code for the previous approach with the same coordinate mesh. This becomes a rather serious disadvantage if the DDCS for the electron energy close to the threshold is computed with the spherically symmetrical coordinate system. Second, this method allows one to compute the DDCS for the electron ejection only in restricted sectors in forward and backward directions.

\section{B. Results of the code implementation}

We have performed the computations for various frequencies of the IR field, but for a fixed delay $t_{\text {delay }}=7$ fs between the center of the XUV attosecond pulse and the onset of the IR pulse. For such a delay, the IR field at the center of the XUV pulse is rather small and the vector potential reaches its extremum, $A_{0}=-0.128$ a.u. In this case the shift of the photoionization spectra by the IR pulse (the streaking effect) is maximal. Within the conventional theory of the streaking effect, the ejected electron energy at the DDCS maximum should be equal approximately to $\epsilon_{\max }=\bar{\epsilon}_{2 p}+\omega_{\mathrm{XUV}}$ $+\sqrt{2\left(\bar{\epsilon}_{2 p}+\omega_{\mathrm{XUV}}\right)} A_{0}+A_{0}^{2}$. The comparison of the maxima of computed DDCS with those given by simple formula is presented in the Table I. The correspondence is very good, thus our code correctly reproduces the streaking effect. However, this effect is rather robust. It appears mainly due to the action of the IR laser field on the ejected electron at large distance. This idea leads to the SFA. It was shown ${ }^{14}$ that SFA nicely reproduce the DDCSs computed in the case of the atom in the gas phase, although the results obtained with the two methods do not coincide completely. Corrections to the crude SFA appear from the contributions to the ejected electron dynamics from quite restricted time and space regions where influences from the IR field and the field of the core and surface are comparable. Study of a deviation of DDCS from the simple model of the streaking effect can be performed only when a concrete experiment is considered.

The correspondence of the computed DDCS with the conventional theory of the streaking effect is much worse in the case of the low frequency $\omega_{\mathrm{XUV}}=45 \mathrm{eV}$. In this case the 
entire structure of DDCS is strongly distorted due to the electron scattering by the surface. This statement is illustrated with Figs. 1(a) and 1(g). The former figure gives the DDCS for the case of the $\mathrm{Na}^{+}$ion adsorbed on the $\mathrm{Ag}(100)$, the later one presents the results of computation for the ionization of this ion in the gas phase. Note that the XUV frequencies are different in these cases. The difference between the frequencies compensates the static shift of the adsorbate ion energy by the image potential $\left(3 / 4 Z_{a t} \approx 8.1 \mathrm{eV}\right)$, so that the energies of the ejected electron just after absorption of the XUV photon are the same in both the cases. Therefore the difference between these figures is due to the electron scattering by the metal surface. Although the XUV frequency $45 \mathrm{eV}$ is out of the frequency range of the current experimental studies, ${ }^{2}$ study of the processes caused by such a pulse could be of interest because at such low frequencies the ejected electrons are slow and the band structure of the metal can play a role in the electron reflection. For slow electrons, the screening of the electron electric field in metals, which leads to the formation of the conventional image charge, is adiabatic. This is assumed within our model. Here it is worthy to note that the attosecond pulse generation for the carrier XUV frequency $\omega_{\mathrm{XUV}}=36 \mathrm{eV}$ was described in Ref. 26. Although this frequency is too small to ionize the $\mathrm{Na}^{+}$adsorbate and the current experimental studies with attosecond pulses are concentrated in the frequency range about $90 \mathrm{eV}$, consideration of the attosecond processes with the carrier frequency in the $40-50 \mathrm{eV}$ range is reasonable. The effect of the conventional polarization shift of the energy of the initial state due to the interaction of the electron with the image charge induced in the metal is clearly seen in Figs. 1(a) and 1(f). Here we are to note that the potential energy by this charge is taken into account in the potential $U_{s}(z)$ within the adiabatic approximation. An improved account of this interaction in the processes with the XUV photon frequency in 90 $\mathrm{eV}$ range requires special study.

One more principal feature of the tasks which can be addressed with this code is scattering of the electrons by the surface. This effect reveals itself with the interference effects. The interference effects are clearly seen in Fig. 1. In Fig. 1(a) this effect leads to the energy gap in the DDCS. In the Figs. 1(b) and 1(c) the interference effect is a relatively weak distortion of DDCS. But in Figs. 1(d)-1(f) the effect is rather strong and becomes qualitative in the shift of the DDCS maximum to nonzero values of the electron ejection angle. The shift increases with the XUV pulse carrier frequency. To make the differences more clear we give Figs. $1(\mathrm{~g})$ and $1(\mathrm{~h})$, which present DDCSs for a solitary $\mathrm{Na}^{+}$ionized with XUV pulses with the frequencies which give the same ejected electron velocity (in absence of the IR pulse) as the XUV pulses in the cases 1a and 1e. The interference is due to the joint action of the potentials and the prompt decrease in the electron wave packet into the metal.

\section{Summary}

A method for numerical simulation of DDCS of electron ejection from an internal shell of an atom adsorbed on a metal surface by attosecond XUV pulse accompanied with
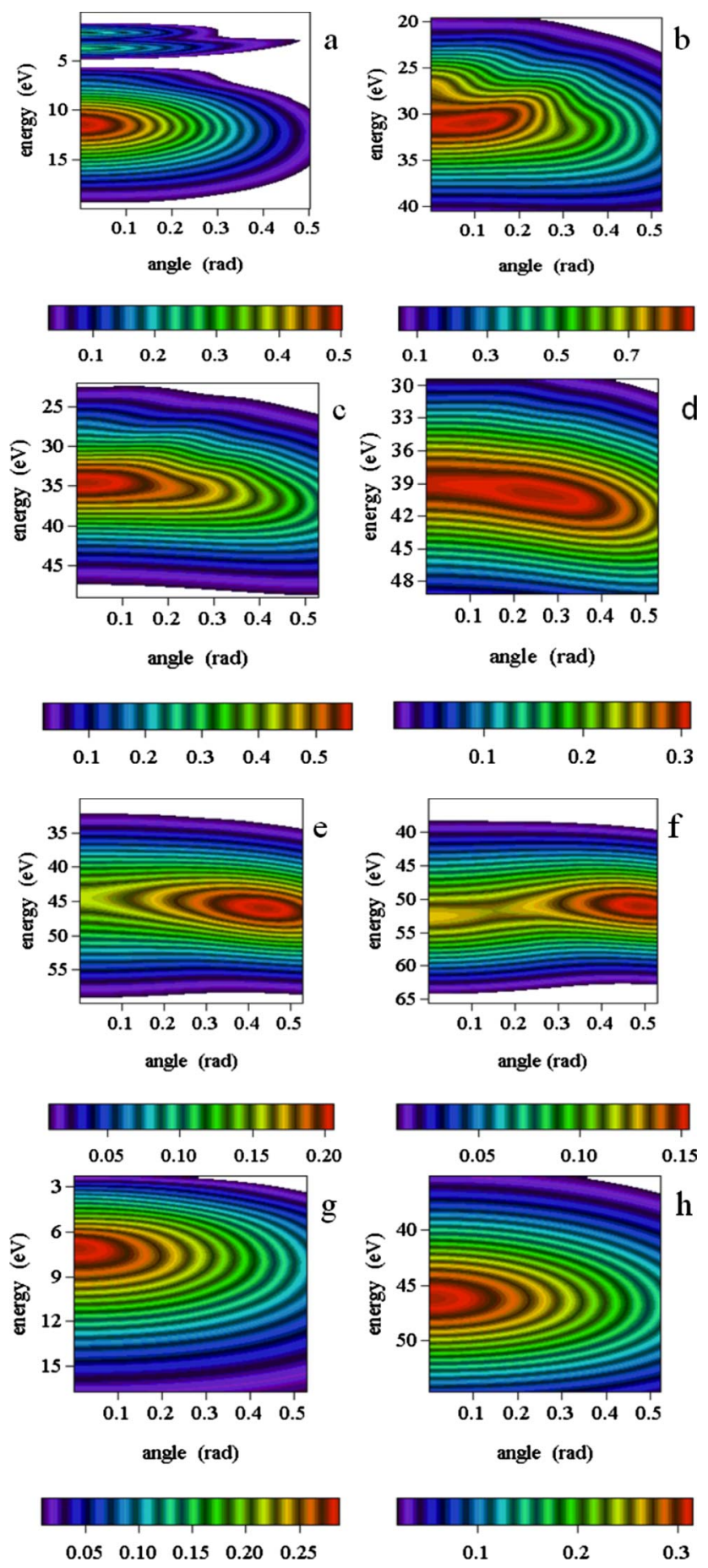

FIG. 1. (Color online) The results of computations of DDCS $d^{2} \sigma / d \epsilon d \Omega$ as functions of the final electron energy $(\epsilon)$ and angle between the final electron momentum and the normal of the surface $(\theta)$ for different frequencies of the XUV pulse $\left(\omega_{\mathrm{XUV}}\right)$. In figures $\mathrm{a}-\mathrm{f}$ the DDCS for ionization of the adsorbate are given and in figures $\mathrm{g}$ and $\mathrm{h}$ the results for ionization of a free $\mathrm{Na}^{+}$ion are plotted. a: $\omega_{\mathrm{XUV}}=45 \mathrm{eV}$; b: $\omega_{\mathrm{XUV}}=75 \mathrm{eV}$; c: $\omega_{\mathrm{XUV}}=80 \mathrm{eV}$; $\mathrm{d}$ : $\omega_{\mathrm{XUV}}=85 \mathrm{eV} ;$ e: $\omega_{\mathrm{XUV}}=90 \mathrm{eV} ;$ f: $\omega_{\mathrm{XUV}}=95 \mathrm{eV} ; \mathrm{g}: \omega_{\mathrm{XUV}}$ $=53.1 \mathrm{eV}$; and h: $\omega_{\mathrm{XUV}}=98.1 \mathrm{eV}$.

strong IR laser field is developed. The implementation of the method has demonstrated that it reproduces the interference effects due to the ejected electron wave-packet scattering by 
the joint action of the core and surface potential, and the streaking effect which appears due to the action on the ejected electron by the laser IR field at large distance. The method can be further developed for the numerical simulation of the effects of relaxation in the electron system of a substrate caused by the abrupt change in the external charge distribution due to absorption of an attosecond pulse. Thus, it can be used both for planning experiments and interpretation of their results.

\section{ACKNOWLEDGMENTS}

A.K.K. deeply acknowledges financial support from the Ikerbasque Foundation. P.M.E. acknowledges partial support from the University of the Basque Country (Grant No. 9/UPV 00206.215-13639/2001), the Basque Universitate eta Ikerketa Saila, and the Spanish Ministerio de Education y Ciencia (MEC) (Grants No. FIS 2004-06490-C03-01 and No. CSD2006-53). We are thankful to F. Krausz, A. G. Borisov, and N. M. Kabachnik for useful discussions.
${ }^{1}$ M. Hentschel, R. Kienberger, Ch. Spielmann, G. A. Reider, N. Milosevic, T. Brabec, P. Corkum, U. Heinzmann, M. Drescher, and F. Krausz, Nature (London) 414, 509 (2001).

${ }^{2}$ P. B. Corkum and F. Krausz, Nat. Phys. 3, 381 (2007).

${ }^{3}$ R. Kienberger, E. Goulielmakis, M. Uiberacker, A. Basltuska, V. Yakovlev, F. Bammer, A. Scrinzi, T. Westerwalbesloh, U. Kleinberger, U. Heinzmann, M. Drescher, and F. Krausz, Nature (London) 427, 817 (2004).

${ }^{4}$ M. Drescher, M. Hentschel, R. Kienberger, M. Uiberacker, V. Yakovlev, A. Scrinizi, T. Westerwalbesloh, U. Kleineberg, U. Heinzmann, and F. Krausz, Nature (London) 419, 803 (2002).

${ }^{5}$ E. Goulielmakis, M. Uiberacker, R. Kienberger, A. Basltuska, V. Yakovlev, A. Scrinzi, T. Westerwalbesloh, U. Kleineberg, U. Heinzmann, M. Drescher, and F. Krausz, Science 305, 1267 (2004).

${ }^{6}$ M. Drescher, M. Hentschel, R. Kienberger, M. Uiberacker, V. Yakovlev, A. Scrinzi, T. Westerwalbesloh, U. Kleineberg, U. Heinzmann, and F. Krausz, J. Electron Spectrosc. Relat. Phenom. 137-140, 259 (2004).

${ }^{7}$ M. Uiberacker, Th. Uphues, M. Schultze, A. J. Verhoef, V. Yakovlev, M. F. Kling, J. Rauschenberger, N. M. Kabachnik, H. Schroder, M. Lezius, K. L. Kompa, H. G. Muller, M. J. J. Vraking, S. Hendel, U. Kleineberg, U. Heinzmann, M. Drescher, and F. Krausz, Nature (London) 446, 627 (2007).

${ }^{8}$ Th. Uphues, M. Schultze, M. F. Kling, M. Uiberacker, S. Hendel, U. Heinzmann, N. M. Kabachnik, and M. Drescher, New J. Phys. 10, 025009 (2008).

${ }^{9}$ A. K. Kazansky and N. M. Kabachnik, J. Phys. B 40, F299 (2007); 41, 135601 (2008).

${ }^{10}$ M. Kitzler, N. Milosevic, A. Scrinzi, F. Krausz, and T. Brabec, Phys. Rev. Lett. 88, 173904 (2002).
${ }^{11}$ J. Itatani, F. Quere, G. L. Yudin, M. Y. Ivanov, F. Krausz, and P. B. Corkum, Phys. Rev. Lett. 88, 173903 (2002).

${ }^{12}$ F. Krausz and M. Ivanov, Rev. Mod. Phys. 81, 163 (2009).

${ }^{13}$ A. L. Cavalieri, N. Muller, T. Uphues, V. Yakovlev, A. Baltuska, B. Horvath, B. Schmidt, L. Blumel, R. Holzwarth, S. Hendel, M. Drescher, U. Kleineberg, P. M. Echenique, R. Kienberger, F. Krausz, and U. Heinzmann, Nature (London) 449, 1029 (2007).

${ }^{14}$ A. K. Kazansky and N. M. Kabachnik, J. Phys. B 39, 5173 (2006); 40, 3413 (2007).

${ }^{15}$ A. K. Kazansky and P. M. Echenique, Phys. Rev. Lett. 102, 177401 (2009).

${ }^{16}$ E. V. Chulkov, V. M. Silkin, and P. M. Echenique, Surf. Sci. 437, 330 (1999).

${ }^{17}$ C. J. Powell and A. Jablonski, J. Phys. Chem. Ref. Data 28, 19 (1999).

${ }^{18}$ V. M. Silkin, private communication.

${ }^{19}$ M. Rocca, F. Biggio, and U. Valbusa, Phys. Rev. B 42, 2835 (1990).

${ }^{20}$ J. D. Jackson, Classical Electrodynamics (John Wiley, New York, 1962).

${ }^{21}$ J. P. Gauyacq, A. G. Borisov, and A. K. Kazansky, J. Phys.: Conf. Ser. 133, 012009 (2008).

${ }^{22}$ A. G. Borisov, A. K. Kazansky, and J. P. Gauyacq, Phys. Rev. B 65, 205414 (2002).

${ }^{23}$ A. K. Kazansky, J. Phys. B 13, L579 (1998).

${ }^{24}$ A. K. Kazansky, A. G. Borisov, and J. P. Gauyacq, Nucl. Instrum. Methods. Phys. B 137, 21 (1999).

${ }^{25}$ J. Sjakste, A. G. Borisov, J. P. Gauyacq, and A. K. Kazansky, J. Phys. B 37, 1593 (2004).

${ }^{26}$ G. Sansone, E. Benedetti, F. Calegari, C. Vozzi, L. Avaldi, R. Flammini, L. Poletto, P. Villoresi, C. Altucci, R. Velotta, S. Stagira, S. De Silvestri, and M. Nisoli, Science 314, 443 (2006) 